\title{
A Comparison between Thermal Buckling Solutions of Power-Law, Sigmoid, Exponential FGM Circular Plates
}

\author{
A. R. Khorshidv and M. R. Eslami
}

\begin{abstract}
In this paper, buckling of elastic, circular plates made of functionally graded material subjected to thermal loading have been investigated. Boundary condition of the plate as immovable clamped edge is considered. The material properties of the FG plates except poisson's ratios are assumed to vary continuously throughout the thickness direction according to the volume fraction of constituents defined by power-law, sigmoid, and exponential function. The Nonlinear equilibrium equations are derived based on the classical plate theory using variational formulations. Linear stability equations are used to obtain the critical buckling of solid FG circular plate under thermal load as uniform temperature rise, linear and nonlinear temperature distribution through the thickness. The effects of P-, S-, E-FGM on buckling of plate are compared. The results are validated with the known data in the literature.
\end{abstract}

Index Terms-Classical plate theory, thermal buckling, functionally graded material.

\section{INTRODUCTION}

Many studies are reported on buckling and bending behavior of FGM structures. Reddy and Khdeir [1] studied the buckling and free-vibration behavior of cross-ply rectangular composite laminated plates using the classical, first order, and third order plate theories under various types of boundary conditions. Exact analytical solutions as well as finite element numerical solutions were developed in their studies. Axisymmetric bending of functionally graded circular and annular plates is studied by Reddy et al. [2]. They presented the solutions for deflections and force and moment resultants based on the first-order plate theory in terms of those obtained using the classical plate theory. The buckling analysis of circular orthotropic and FGM plates under thermal loads is given by Najafizadeh, and Eslami [3]-[4]. Khorshidvand et al. [5] presented buckling analysis of circular FGM plate integrated with piezoelectric layers subjected to three kinds of thermal loadings based on classical plate theory. Saidi and Hasani Baferani [6] studied the thermal buckling analysis of moderately thick functionally graded annular sector plate based on the first order deformation plate theory by using the equilibrium and stability equation.

Khorshidvand et al. [7] presented the thermal and

Manuscript received August 15, 2012; revised November 14, 2012. This work was supported in part by Department of Mechanical Engineering, South Tehran Branch, I. A.U.

A. R. Khorshidvand is with the engineering Faculty, Department of Mechanical Engineering, South Tehran Branch, Islamic Azad University, Tehran, Iran (e-mail: AR_Khorshidvand@azad.ac.ir).

M. R. Eslami is with the Mechanical Engineering Department, Amirkabir University of Technology, Tehran 15914, Iran (e-mail: eslami@aut.ac.ir). mechanical stability problem of circular hybrid FGM plates based on first order shear deformation plate theory and derived closed-form solutions for critical buckling temperatures of perfect piezoelectric functionally graded plates, which are subjected to thermal and mechanical loadings and applied constant voltage.

The present paper deals with determination of the stability problem and presents closed-form solutions for critical buckling temperature of P-, S-, E-FGM circular plate, which are subjected to three kinds of thermal loading. Clamped edge boundary condition is assumed for the plate.

\section{PROCEDURE FOR PAPER SUBMISSION}

Consider a uniform thin circular plate made of FGM, as shown in Fig. 1. To extract formulations, a cylindrical coordinate system is taken in the center of plate's middle plane. The FGM profile across the thickness direction of the plate, made of ceramic and metal constituent materials, may be assumed to follow a function form as P-FGM plates as

$$
\operatorname{Pr}(z)=P r_{m}+P r_{c m}\left(\frac{2 z+h}{2 h}\right)^{n}
$$

The value of $n$, power law index, equal to zero represents a fully ceramic plate. Two power law functions S-FGM plates as

$$
\begin{array}{lll}
\operatorname{Pr}(z)=P r_{m}+\operatorname{Pr}_{c m}\left[1-\frac{1}{2}\left(\frac{h-2 z}{h}\right)^{n}\right] & \text { for } & 0 \leq z \leq h / 2 \\
\operatorname{Pr}(z)=P r_{m}+P r_{c m}\left[\frac{1}{2}\left(\frac{h+2 z}{h}\right)^{n}\right] & \text { for } & -h / 2 \leq z \leq 0
\end{array}
$$

and exponential function E-FGM plates

$$
\operatorname{Pr}(z)=A \operatorname{Exp}\left[B\left(\frac{h+2 z}{2 h}\right)\right], \quad A=P r_{m}, \quad B=\operatorname{Ln}\left(P r_{c} / P r_{m}\right)
$$

where $\mathrm{pr}_{\mathrm{p}} \mathrm{pr}_{\mathrm{c}}, \mathrm{pr}_{\mathrm{m}}$ denote any material property of the FGM, metal, and ceramic; such as the modulus of elasticity $E$ and the coefficient of thermal expansion $\alpha$. Note that the volume fraction of the ceramic is high near the top surface of the plate, and that of metal is high near the bottom surface. The relations (1), (2) and (3) indicate that the top surface of the plate $(z=h / 2)$ is ceramic-rich whereas the bottom surface $(z=-h / 2)$ of the plate is metal-rich. Generally, Poisson's ratio is assumed constant across the plate thickness.

The material properties are assumed to be independent of temperature, and the stress and strain relations are linear. The constitutive relations of functionally graded materials in thermal environment for the plane-stress condition are written as

$$
\sigma_{r r}=E(z)\left(\varepsilon_{r r}+v \varepsilon_{\theta \theta}\right) /\left(1-v^{2}\right)-E(z) \alpha(z) T(z) /(1-v)
$$


$\sigma_{\theta \theta}=E(z)\left(\varepsilon_{\theta \theta}+v \varepsilon_{r r}\right) /\left(1-v^{2}\right)-E(z) \alpha(z) T(z) /(1-v)$

$$
\sigma_{r \theta}=E(z) /(1+v) \varepsilon_{r \theta}
$$

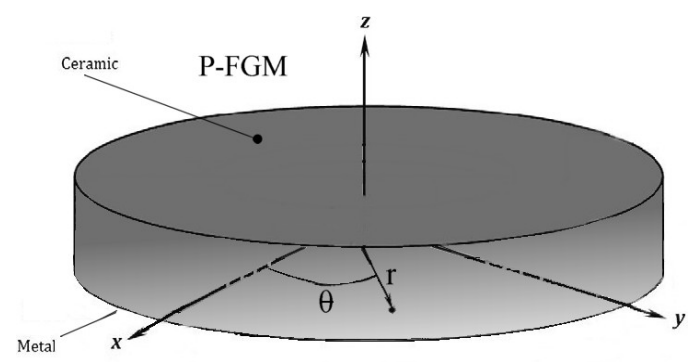

Fig. 1. Geometry of FG circular plate

The plate is assumed to be comparatively thin, and according to the Love-Kirchhoff assumptions, shear deformations normal to the plate are disregarded. Using the classical plate theory (CPT), strain components at distance $\mathrm{z}$ from the middle plane are given in matrix form as [8]

$$
\begin{aligned}
& \left\{\begin{array}{c}
\varepsilon_{r r} \\
\varepsilon_{\theta \theta} \\
\gamma_{r \theta}
\end{array}\right\}=\left\{\begin{array}{c}
u_{, r}+\frac{1}{2}\left(w_{, r}\right)^{2} \\
\frac{1}{r} v_{,}+\frac{1}{r} u+\frac{1}{2}\left(\frac{1}{r} w_{, \theta}\right)^{2} \\
\frac{1}{r} u_{, \theta}+v_{, \theta}-\frac{1}{r} v_{,}+\frac{1}{2}\left(w_{, \theta}\right)^{2}
\end{array}\right\}+z\left\{\begin{array}{c}
-w_{, r r} \\
-\left(\frac{1}{r} w_{, r}+\frac{1}{r^{2}} w_{, \theta \theta}\right) \\
-\frac{1}{r} w_{, r}+\frac{1}{r^{2}} w_{, \theta}
\end{array}\right\} \\
& \varepsilon=\varepsilon^{(0)}+\mathrm{Z} \varepsilon^{(1)}
\end{aligned}
$$

where a comma in subscript indicates partial differentiation and where $\varepsilon_{r r}, \varepsilon_{\theta \theta}$, and $\gamma_{r \theta}$ are the normal and shear strain components along the r-, $\theta$ - respectively. The stress components in plane-stress condition in the plate (superscript h) are written as following

$$
\{\sigma\}^{h}=\left\{\begin{array}{c}
\sigma_{r r} \\
\sigma_{\theta \theta} \\
\sigma_{r \theta}
\end{array}\right\}^{h}=\left[\begin{array}{ccc}
Q_{11} & Q_{12} & 0 \\
Q_{21} & Q_{22} & 0 \\
0 & 0 & Q_{44}
\end{array}\right]\left\{\begin{array}{c}
\varepsilon_{r r}-\alpha(z) T(z) \\
\varepsilon_{\theta \theta^{-\alpha(z) T(z)}} \\
\gamma_{r \theta}
\end{array}\right\}
$$

where the plane-stress-reduced stiffness are defined as

$$
Q_{11}=Q_{22}=\frac{E(z)}{1-v^{2}}, \quad Q_{12}=Q_{21}=v Q_{11}, \quad Q_{44}=\frac{E(z)}{2(1+v)}
$$

The total potential energy for FG circular plate can be written as follows

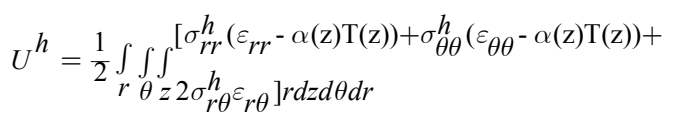

Considering relations (4) to (9) and substituting them into relation (10) and integrating with respect to $z$, the total potential energy is obtained. Applying the Euler equations for total functional of $U$ in Eq. (10), equilibrium equations yield [8]

$$
\begin{gathered}
\left(r N_{r r} w_{1, r}+N_{r \theta^{w}, \theta}-M_{\theta \theta}\right)_{, r}+\left(\frac{1}{r} N_{\theta \theta^{w}, \theta}+N_{r \theta}{ }_{, r}\right. \\
\left.+\frac{2}{r} M_{r \theta}\right)_{, \theta}+\left(r M_{r r}\right)_{, r r}+\left(2 M_{r \theta}\right)_{, r \theta}+\left(\frac{1}{r} M_{\theta \theta}\right)_{, \theta \theta}=0 \\
N_{r r, r}+\frac{1}{r}\left(N_{r r}-N_{\theta \theta}\right)+\frac{1}{r} N_{r \theta, \theta}=0
\end{gathered}
$$

$$
\frac{2}{r} N_{r \theta}+\frac{1}{r} N_{\theta \theta, \theta}+N_{r \theta, r}=0
$$

The stability equations of the circular plate are derived using the adjacent equilibrium criterion. We assume $u_{0}, v_{0}$, and $w_{0}$ as the displacement components of the equilibrium state and $u_{1}, v_{1}$, and $w_{1}$ as the virtual displacements corresponding to a neighboring state. The displacement components and then the linear incremental stress resultants are

$$
\begin{gathered}
u=u_{0}+u_{1} \quad v=v_{0}+v_{1} \quad w=w_{0}+w_{1} \\
N_{r r}=N_{r r 0}+N_{r r 1} M_{r r}=M_{r r 0}+M_{r r 1} \\
N_{\theta \theta}=N_{\theta \theta 0}+N_{\theta \theta 1} M_{\theta \theta}=M_{\theta \theta 0}+M_{\theta \theta 1} \\
N_{r \theta}=N_{r \theta 0}+N_{r \theta 1} M_{r \theta}=M_{r \theta 0}+M_{r \theta 1}
\end{gathered}
$$

Substituting relations (12) in Eqs. (11) and collecting the second order terms, the stability equations are obtained as

$$
\begin{gathered}
\left(r N_{r r 1} w_{1, r}+N_{r \theta 1} w_{1, \theta}-M_{\theta \theta 1}\right)_{, r}+\left(\frac{1}{r} N_{\theta \theta 1} w_{1, \theta}+N_{r \theta 1} w_{1, r}\right. \\
\left.+\frac{2}{r} M_{r \theta 1}\right)_{, \theta}+\left(r M_{r r 1}\right)_{, r r}+\left(2 M_{r \theta 1}\right)_{, r \theta}+\left(\frac{1}{r} M_{\theta \theta 1}\right)_{, \theta \theta}=0 \\
N_{r r 1, r}+\frac{1}{r}\left(N_{r r 1}-N_{\theta \theta 1}\right)+\frac{1}{r} N_{r \theta 1, \theta}=0 \\
\frac{2}{r} N_{r \theta 1}+\frac{1}{r} N_{\theta \theta 1, \theta}+N_{r \theta 1, r}=0
\end{gathered}
$$

The force and moment resultants of plate are expressed in terms of the stress components through the thickness as follow

$$
\begin{gathered}
\{N\}=\left\{\begin{array}{lll}
N_{r r} & N_{\theta \theta} & N_{r \theta}
\end{array}\right\}^{T}=\int_{-h / 2}^{h / 2}\{\sigma\}^{h} d z \\
\{M\}=\left\{\begin{array}{lll}
M_{r r} & M_{\theta \theta} & \left.M_{r \theta}\right\}^{T}=\int_{-h / 2}^{h / 2}\{\sigma\}^{h} z d z
\end{array}\right.
\end{gathered}
$$

Stress resultants can be simplified in the matrix form as

$$
\left\{\begin{array}{l}
\{N\} \\
\{M\}
\end{array}\right\}=\left[\begin{array}{ll}
{[A]} & {[B]} \\
{[B]} & {[D]}
\end{array}\right]\left\{\begin{array}{c}
\varepsilon^{(0)} \\
\varepsilon^{(1)}
\end{array}\right\}-\left\{\begin{array}{c}
\left\{N^{(T)}\right\} \\
\left\{M^{(T)}\right\}
\end{array}\right\}
$$

where

$$
\left(A_{i j}, B_{i j}, D_{i j}\right)=\int_{z} Q_{i j}(z)\left(1, z, z^{2}\right) d z \quad(i, j=1,2,3)
$$

Here $\left\{N^{(T)}\right\},\left\{M^{(T)}\right\}$ are the stress resultants due to the applied temperature field on the plate, and they can be computed as

$$
\begin{aligned}
& \left\{N^{(T)}\right\}_{F G M}=\left\{\begin{array}{lll}
E_{4} /(1-v) & E_{4} /(1-v) & 0
\end{array}\right\}^{T} \\
& \left\{M^{(T)}\right\}_{F G M}=\left\{\begin{array}{lll}
E_{5} /(1-v) & E_{5} /(1-v) & 0
\end{array}\right\}^{T}
\end{aligned}
$$

where

$$
E_{4}=\int_{-h / 2}^{h / 2} \alpha(z) E(z) \Delta T d z \quad, \quad E_{5}=\int_{-h / 2}^{h / 2} z \alpha(z) E(z) \Delta T d z
$$

Here polar symmetry condition is considered. Thus, for this case of discussion the first and second of stability 
equations (13), based on the displacement components, lead to

$$
\begin{array}{r}
E_{3}^{*} \nabla^{4} w_{1}+N_{r r 0} w_{1}{ }^{\prime \prime}+\frac{1}{r} N_{\theta \theta 0} w_{1}{ }^{\prime}+E_{2}^{*}\left(-u_{1}{ }^{\prime \prime}-\frac{2}{r} u_{1}{ }^{\prime \prime}+\frac{1}{r^{2}} u_{1}{ }^{\prime}-\frac{1}{r^{3}} u_{1}\right)=0 \\
E_{1}^{*}\left(u_{1}{ }^{\prime \prime}+\frac{1}{r} u_{1}^{\prime}-\frac{1}{r^{2}} u_{1}\right)+E_{2}^{*}\left(-w_{1}^{\prime \prime \prime}-\frac{1}{r} w_{1}^{\prime \prime}+\frac{1}{r^{2}} w_{1}^{\prime}\right)=0(19)
\end{array}
$$

where $E_{1}^{*}, E_{2}^{*}, E_{3}^{*}$ are given as

$$
\left(E_{1}^{*}, E_{2}^{*}, E_{3}^{*}\right)=1 /\left(1-\nu^{2}\right) \int_{-h / 2}^{h / 2}\left(1, z, z^{2}\right) E(z) d z
$$

Referring to Eqs. (15), using the membrane plate theory, the prebuckling forces are obtained as

$$
N_{r r 0}=-N_{r r 0}^{(T)}, N_{\theta \theta 0}=-N_{\theta \theta 0}^{(T)}
$$

Thus, the set of coupled stability equations must be solved. For clamped and immovable edge in $r$ - direction, the boundary conditions are expressed as [9]

$$
\begin{gathered}
u_{1}(r=0)=0, \quad w_{1}(r=0)=\text { finite } \\
u_{1}(r=a)=w_{1}(r=a)=w_{1}^{\prime}(r=a)=0
\end{gathered}
$$

The solution of Eqs. (19) is assumed in the form

$$
\begin{aligned}
& u_{1}(r)=A_{1} J_{1}(\lambda r)+A_{2} Y_{1}(\lambda r)+A_{3}(1 / r)+A_{4} r \\
& w_{1}(r)=A_{5} J_{0}(\lambda r)+A_{6} Y_{0}(\lambda r)+A_{7} L n r+A_{8}
\end{aligned}
$$

where $J_{0}, J_{1}$ and $Y_{0}, Y_{1}$ are the Bessel functions of first, zero order, and first and second kinds, respectively. Also, $A_{1}$ to $A_{8}$ are the integration constants. Using the first and second boundary conditions yields $A_{2}=A_{3}=A_{6}=A_{7}=0$. Satisfying the third boundary condition of Eqs. (22),

$$
A_{4}=0, \quad A_{8}=-J_{0}(\lambda a) A_{5}, \quad-J_{1}(\lambda a)=0
$$

Thus, the smallest root is $\lambda \mathrm{a}=3.83$. It is seen that for the clamped edge

$$
u_{1}(r)=A_{1} J_{1}(\lambda r), w_{1}(r)=A_{5}\left(J_{0}(\lambda r)-J_{0}(\lambda a)\right)
$$

Substituting the expressions (25) into (23), two linear homogeneous equations are obtained as

$$
\begin{gathered}
-\lambda^{2} E_{1}^{*} A_{1}-\lambda^{3} E_{2}^{*} A_{5}=0 \\
\lambda^{3} E_{2}^{*} A_{1}+\left[\lambda^{4} E_{3}^{*}-\lambda^{2} N_{r r 0}^{(T)}\right] A_{5}=0
\end{gathered}
$$

For a nontrivial solution of these equations, the determinant of coefficient must be set to zero and when the temperature distribution of the plate is a function of thickness direction only, $\lambda$ is constant and yields.

$$
\lambda^{2}=N_{r r 0}^{(T)} /\left[E_{3}^{*}-E_{2}^{* 2} / E_{1}^{*}\right]
$$

Now, for the case of uniform temperature rise, consider a plate at temperature $T_{0}$, the temperature may be raised to $T_{f}$ where the plate buckles. In such a case, using Eq. (27) and taking relation (28) the critical buckling temperature $\Delta T_{c r}$ is expressed in the form

$$
\Delta T_{c r}=(1-v)\left\{\lambda^{2}\left(E_{3}^{*}-E_{2}^{* 2} / E_{1}^{*}\right)\right\} / Q_{1}
$$

where

$$
Q_{1}=\left[\alpha_{m} E_{m}+\frac{1}{(1+n)}\left(\alpha_{m} E_{c m}+\alpha_{c m} E_{m}\right)+\frac{1}{(2 n+1)} \alpha_{c m} E_{c m}\right] h
$$

$$
\Delta T_{c r}=T_{f}-T_{0} \text {. For thin circular plates, the temperature }
$$
distribution may be assumed linear through the thickness of the plate as

$$
T(z)=\Delta T(z+h / 2) / h+T_{1}
$$

Consider temperature $T_{1}$ at top side of the plate and $T_{2}$ at the bottom side. The critical value of temperature difference is calculated to be

$$
\Delta T_{c r}=\frac{(1-v)}{\left(0.5 Q_{1}+Q_{2}\right)}\left\{-\frac{T_{1} Q_{1}}{(1-v)}+\lambda^{2}\left(E_{3}^{*}-E_{2}^{* 2} / E_{1}^{*}\right)\right\}
$$

where

$$
Q_{2}=\int_{z} z \alpha(z) E(z) d z
$$

To calculate the critical buckling temperature for the case of gradient through the thickness of plate, the one-dimensional equation of heat conduction in the $\mathrm{z}$ direction must be solved. The heat conduction equation for the steady state one-dimensional case, in the absence of heat generation, becomes

$$
\frac{d}{d z}\left(K(z) \frac{d T}{d z}\right)=0
$$

Temperature boundary conditions at the top and bottom surfaces of the plate are

$$
T\left(\frac{h}{2}\right)=T_{c} \quad, \quad T\left(-\frac{h}{2}\right)=T_{m}
$$

The solution of the heat conduction equation along with the thermal boundary conditions is obtained via the power-series solution as

$$
T(z)=T_{m}+\left(T_{c}-T_{m}\right) \frac{\sum_{i=0}^{N} \frac{1}{i n+1}\left(-\frac{K_{c m}}{K_{m}}\right)^{i}\left(\frac{1}{2}+\frac{z}{h}\right)^{i n+1}}{\sum_{i=0}^{N} \frac{1}{i n+1}\left(-\frac{K_{c m}}{K_{m}}\right)^{i}}
$$

where $N$ is the number of expanded terms and should be chosen to assure the convergence of the solution.

Hence the critical temperature difference is

$$
\Delta T_{c r}=(1-v)\left\{\left(-T_{2}-T_{0}\right) Q_{1}+\lambda^{2}\left(E_{3}^{*}-E_{2}^{* 2} / E_{1}^{*}\right)\right\} / Q_{3}
$$

in which

$$
\begin{aligned}
& Q_{3}=\left\{E_{m} \alpha_{m} \sum_{i=0}^{N} \frac{1}{(i n+1)(i n+2)}\left(-\frac{K_{c m}}{K_{m}}\right)^{i}\right. \\
& +\left(E_{m} \alpha_{m}+E_{m} \alpha_{c m}\right) \sum_{i=0}^{N} \frac{1}{(i n+1)(i n+n+2)}\left(-\frac{K_{c m}}{K_{m}}\right)^{i}
\end{aligned}
$$

$$
\left.+E_{c m} \alpha_{c m} \sum_{i=0}^{N} \frac{1}{(i n+1)(i n+2 n+2)}\left(-\frac{K_{c m}}{K_{m}}\right)^{i}\right\}
$$


TABLE I: MATERIAl Properties of Plate

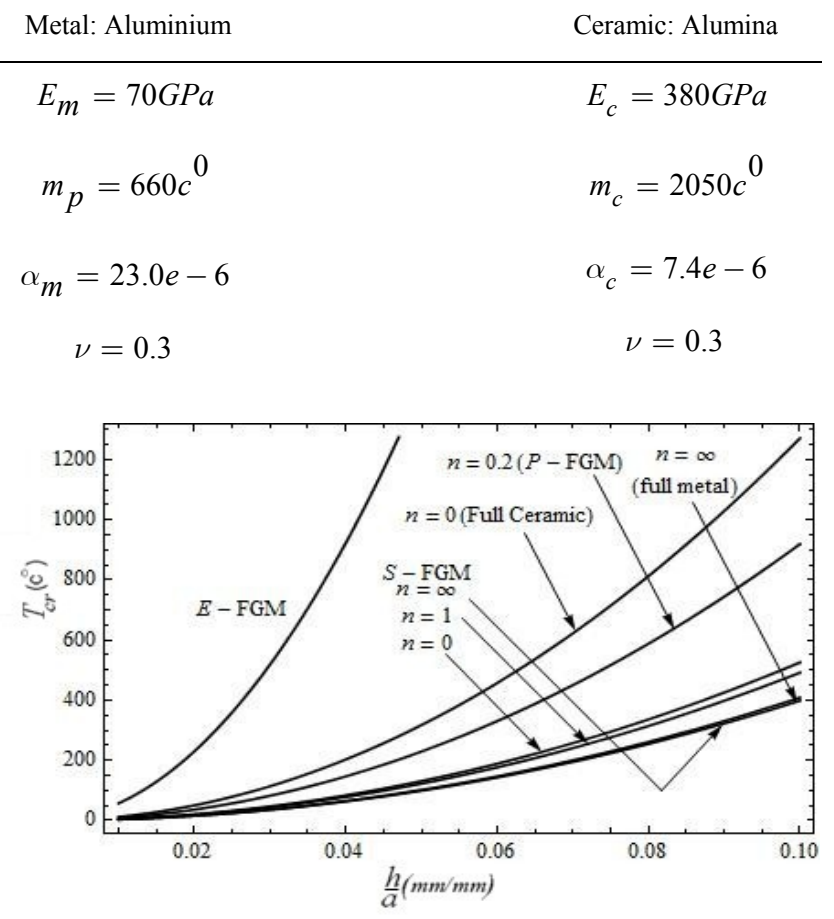

Fig. 2. Buckling temperature versus h/a for a P-,S-,E-FGM circular clamped plate under uniform temperature rise.

\section{RESULTS AND DISCUSSION}

In the following, the axisymmetric stability and thermal buckling loads of an FG circular plate subjected to uniform temperature rise is derived and summarized in the preceding section. To validate the formulations of the present article, thermal buckling loads of the circular plate are compared with those obtained by Najafizadeh and Eslami [2] for isotropic plate. It is clear that from Eq. (27), taking PFGM circular plate, the same results is obtained for the homogeneous isotropic full ceramic circular plate. The results are obtained that are identical to those reported as in [2]. Now, consider an FGM circular plate. The material properties of metal (Aluminium), and ceramic (Alumina) constituents are given in Table I. For this example the results for thermal buckling loads is plotted in Fig. 2. Fig. 2 represents the critical buckling temperature versus $\mathrm{h} / \mathrm{a}$ for a P-, S-, E-FGM clamped circular plate under uniform temperature rise. The mechanical boundary condition at the edge of the plate is assumed to be clamped supported. Here, the effect of FGM models and volume fraction index on thermal bucking temperature of plate can be seen.

\section{CONCLUSION}

In the present article, the buckling analysis of FG circular plate is derived based on classical plate theory with the assumption of power law, Sigmoid, Exponential composition for the constituent materials. Boundary condition of the plate is taken to be clamped. Plate is subjected to uniform temperature rise, linear and nonlinear temperature distribution through the thickness. The thermal buckling capacity of circular plates as closed-form solution is presented.

It is concluded that:

1) The equilibrium and stability equations are identical for P-, S-, E-FGM plates.

2) The critical buckling temperature is reduced when volume fraction index increases, as the plate becomes more metal-rich.

3) A comparison between thermal buckling curves of power-law, sigmoid, exponential FGM circular plates in uniform temperature rise, indicate that for a certain value of $h / a$ thermal buckling capacity of circular plate made of P-FGM is better than S-FGM.

\section{ACKNOWLEDGMENT}

The present research work was supported by Islamic Azad University - South Tehran Branch.

\section{REFERENCES}

[1] J. N. Reddy and A. A. Khdeir, "Buckling and vibration of laminated composite plate using various plate theories," AIAA Journal, vol. 27, no. 12, pp. 1808-1817, 1989.

[2] J. N. Reddy, C. M. Wang, and S. Kitipornchai, "Axisymmetric bending of functionally graded circular and annular plates," Eur. J. Mech. A/solids, no. 18, pp. 195-99, 1999.

[3] M. M. Najafizadeh and M. R. Eslami, "Thermoelastic stability of orthotropic circular plates," Journal of Thermal Stresses, vol. 25, no. 10, p. $985-1005,2002$

[4] M. M. Najafizadeh and M. R. Eslami, "Thermoelastic stability of circular plates composed of functionally graded materials based on first order theory," AIAA Journal, vol. 40, no. 7, pp. 1444-1450, 2002.

[5] A. R. Khorshidvand, M. Jabbari, and M. R. Eslami, "Thermoelastic buckling analysis of functionally graded circular plates integrated with piezoelectric layers," Journal of Thermal Stresses, vol. 35, pp. 695-717, 2012.

[6] A. R. Saidi and A. H. Baferani, "Thermal buckling analysis of moderately thick functionally graded annular sector plates," Composite Structures, vol. 92, no. 7, pp. 1744-1752, 2010.

[7] A. R. Khorshidvand, Y. Kiani, M. Jabbari, and M. R. Eslami, "Thermo-electro-mechanical Buckling of Shear Deformable Hybrid Circular FGM Plates," Journal of Mechanics of Advanced Materials and Structures (submitted for publication).

[8] D. O. Brush and B. O. Almorth, Buckling of bars, plates and shells. McGraw-Hill, New York, 1975.

[9] J. N. Reddy, Mechanics of laminated composite plates and shells, theory and analysis. CRC Press, New York, 2004.

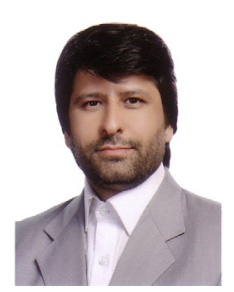

Ahmad Reza Khorshidvand was born in Tehran, Iran, in 1969. He received $\mathrm{PhD}$ degree in mechanica engineering from Science and Research Branch, IAU, Tehran, IRAN. Now, he is member of faculty of mechanical engineering department, South Tehran Branch, Tehran, IRAN. His research interests include Solid Mechanics, Thermo-Elasticity, Composite Materials, and Stability. Dr. Khorshidvand is member of IACSIT and he has more than six published papers in International Journals. 